\title{
NOTES
}

\section{Small Angle Light Scattering Studies on Bisphenol Series Thermotropic Liquid Crystalline Wholly Aromatic Polymers}

\author{
Zhouming ZHAO* and Xuqin Wu \\ Department of Polymer Material Science and Engineering, \\ East China University of Chemical Technology, \\ Shanghai 200237, People's Republic of China
}

(Received November 9, 1993)

\begin{abstract}
KEY WORDS Liquid Crystalline / Bisphenol / SALS / Sheared Mesophase / Domain Sizes / Rod-Like Units /
\end{abstract}

Thermotropic liquid crystalline polymers (TLCPs) offer great potential for producing materials with superior mechanical properties because of the anisotropic melt mesophase. The anisotropic properties have been studied on many TLCPs in fields of morphology, rheology, and rheo-optics, et al. ${ }^{1-5}$ During these studies, small angle light scattering (SALS) has been frequently used and been particularly useful in characterizing supermolecular structures, optical anisotropy and the average domain sizes.

In previous studies, ${ }^{6}$ a family of thermotropic liquid crystalline aromatic copolyesters were prepared based on terephthalic acid (TPA), isophthalic acid (IPA), $p$-hydroxybenzoic acid (HBA) and one of the following bisphenols: 4,4'-dihydroxydiphenyl (BP), 4,4'dihydroxydiphenyl methane (BPM), 4,4'-dihydroxydiphenyl propane (BPA), or 4,4'dihydroxydiphenyl sulfone (SDP). The mole ratio of HBA/bisphenol/TPA/IPA in the reaction mixture was 60/20/14/6. The correspondent copolyesters were termed TIHBP, TIHBPM, TIHBPA, and TIHSDP. The copolyesters exhibited Schlieren nematic mes- ophase textures within broad temperature ranges $\left(>70^{\circ} \mathrm{C}\right)$. Azimuthally independent SALS patterns were reported for the undeformed nematic mesophases, which was one type of the typical scattering patterns for rod-like scattering units. ${ }^{7}$ The deformation of the melts in mesophase was also qualitatively discussed by using polarized optical microscopy and SALS.

In the present work, the SALS technique was used to investigate the average domain sizes in sheared nematic mesophase for this family of aromatic copolyesters. A consistent result was obtained between the steric effect of the bridge groups in the bisphenols and the domain sizes, which, to the author's knowledge, has never been published before.

\section{EXPERIMENTAL}

Since the copolyesters did not dissolve in any solvent, the solution-casting method could not be used to make sample film. The materials were first ground into fine powders and extracted in a Soxhlet with acetone for $24 \mathrm{~h}$, and then dried in a vacuum oven for $8 \mathrm{~h}$.

* Present address: Department of Chemistry and Chemical Engineering, Box 63, Stevens Institute of Technology, New Jersey 07306, U.S.A. 
A small amount of the polymer sample was placed between two microscope cover glasses to form a sandwich. The sandwich was then compressed in a special holder on a stage equipped with micrometer screw caliper to form a specimen with thickness of $c a .20 \mu \mathrm{m}$. The sandwich was then put on a hot-stage which had been preheated to a temperature of $40^{\circ} \mathrm{C}$ above the polymer melting point $T_{\mathrm{m}}$, which was $331.0,285.8,310.0$, and $291.9^{\circ} \mathrm{C}$ for TIHBP, TIHBPM, TIHBPA, and TIHSDP, respectively. The completely melted sample was sheared between the two glass slides at a shear rate on the order of $10^{2} \mathrm{~s}^{-1}$. Within $1 \mathrm{~s}$, the deformed melt film was transferred to an ice-water bath and quenched for $30 \mathrm{~s}$. The bath temperature was well below the glass transition temperatures $\left(T_{\mathrm{g}} \mathrm{s}\right)$ of those rigid chain copolyesters, which were 212.3, 95.7, 112.3 , and $168.3^{\circ} \mathrm{C}$ for TIHBP, TIHBPM, TIHBPA, and TIHSDP, respectively. The melt mesophases were therefore frozen and could not be relaxed even at the room temperature. The thickness of the quenched melt film was ca. $15 \mu \mathrm{m}$, when measured on the compress stage. The SALS experiments of the samples were performed by using a SP SALS-1 Small Angle Light Scattering apparatus, equipped with a $\mathrm{He}-\mathrm{Ne}$ laser source with a wavelength of $632.8 \mathrm{~nm}$ on, an optical bench with a pair of crossed polarizers, and an optical detector system. A 1-mm pinhole was placed behind the sample. The $H_{\mathrm{v}}$ scattering patterns under crossed polarizers were photographed by a camera. The sample-to-film distance was $40 \mathrm{~cm}$. The light intensity was calibrated with a standard quartz. All the measurements were carried out at $20 \pm 0.5^{\circ} \mathrm{C}$.

\section{RESULTS AND DISCUSSION}

Figure 1 was the $H_{\mathrm{v}}$ SALS pattern of the nematic mesophase after melt shear deformation for copolyester TIHBP, which was in a two-leaf clover shape with lobes oriented along the horizontal direction, i.e., having a maxi-

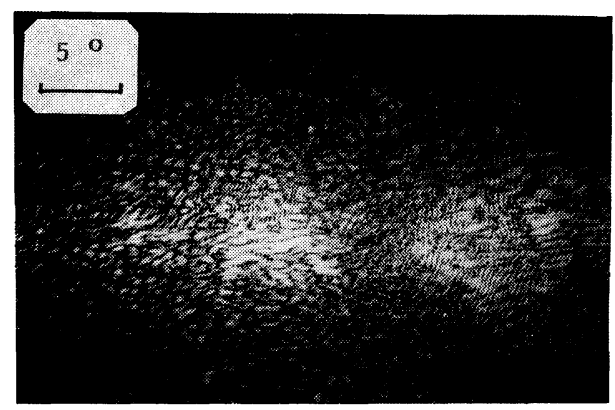

(a)

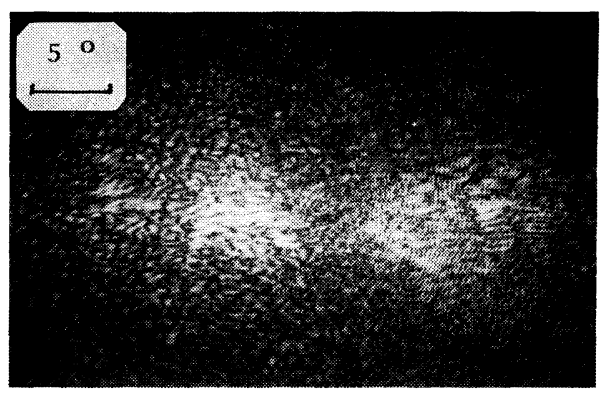

(b)

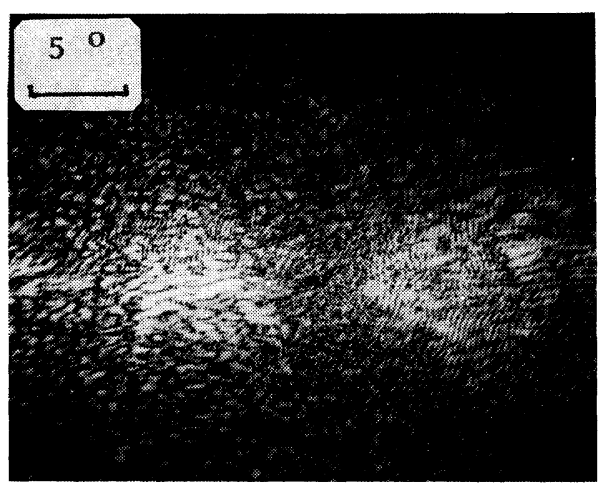

(c)

Figure 1. $H_{\mathrm{v}}$ SALS patterns for the copolyester TIHBP, shear was along the vertical direction of $X$ axis.

mum light intensity at azimuthal angles $(u)$ of $0^{\circ}$ and $180^{\circ}$. Similar SALS patterns were also found for the copolyesters TIHBPM, TIHSDP, and TIHBPA. Usually, either circular or four-leaf clover patterns were observed for unoriented nematic mesophases. A polymer's SALS pattern could be, in fact, influenced by 
different factors such as polymer's structure, thickness, molecular weight, sample preparation, annealing time which could lead to different superstructures, et al..$^{1,2,7-11)}$ Here, the two-leaf clover patterns were based on the shear-oriented samples. The patterns were not so common as most of the four-leaf clover patterns, either $\times$ type or + type, which had been simulated and calculated using different structure models for the situations arising from the scattering of anisotropic rods. ${ }^{12-16}$ Generally speaking, those models could be separated into two types: a) isolated system and b) correlated system. As a representative of the former, Kawai et al. ${ }^{17}$ developed a model based on assumption that the scattering pattern was calculated for a single isolated rod without considering the interactions between the rods. This model could provide simple calculation equations for different scattering patterns. Only the length of the rod was of consequence in the model. On the other hand, Hashimoto et al. ${ }^{18}$ proposed a model for the domain structure of a liquid crystalline polymer considering the main-body interactions of disclinations. However, the method was not so simple to interpret and the average domain size cannot be directly calculated as by using the Kawai's method for the optically anisotropic rods. Both Kawai's model and Hashimoto's system were based on the assumption of random assembly of anisotropic rods. Moreover, the liquid crystalline polymers used here had the preferred orientation due to the shear deformation. Thus neither Kawai's model nor Hashimoto's system could be well suitable for the situation here in a satisfying way. As an attempt to quantitatively characterize the domain spacing, we took Kawai's model which provided an adequate calculation basis for our case. For the + type fourfold symmetric pattern in Kawai's model, the rod length $L$ could be calculated by eq 1 :

$$
4.8=(2 L / \lambda) \sin \left(\theta_{\max } / 2\right)
$$

where $\lambda$ was the wavelength of light beam in

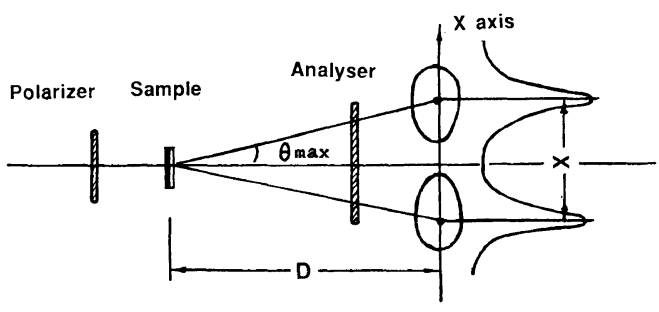

Figure 2. Experimental method for measuring $\theta_{\max }$.

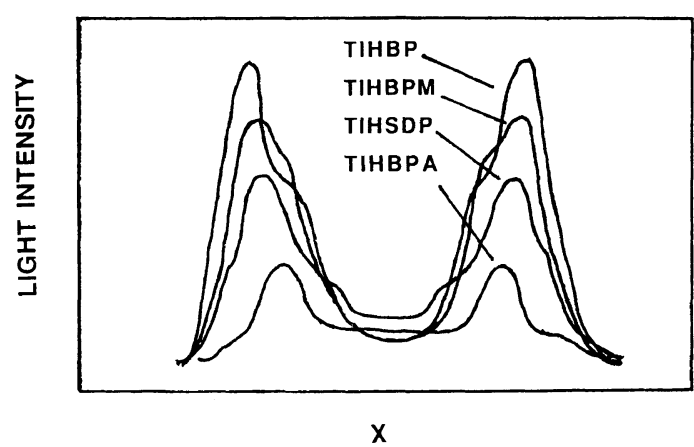

Figure 3. The distribution of scattering light intensity along $X$ axis.

the medium and the $\theta_{\max }$ was the scattering angle at the scattering intensity maxima. The two-leaf clover $H_{\mathrm{v}}$ patterns in Figure 1 could be treated as a special case of the +type fourfold pattern with zero scattering intensity at azimuthal angles $u=90^{\circ}$ and $u=270^{\circ}$. Then the rod length in the sheared mesophase could be obtained by measuring the maximum scattering intensity at a scattering angle $\theta=\theta_{\max }$ occurring at the azimuthal angle $u_{\max }$ along $X$ axis, or $u=0^{\circ}$ and $u=180^{\circ}$. Here, $\theta_{\max }$ could be experimentally determined from eq 2 as illustrated in Figure 2. The domain sizes, as listed in Table I, were then

$$
\theta_{\max }=\operatorname{tg}^{-1}(X / 2 D)
$$

calculated by combining eq 1 and 2 based on the light intensity measurements in Figure 3.

In Schlieren textures, since the length of rod was determined by the average disclination density, the size parameter would in fact be referred to as an "average domain size". ${ }^{18}$ The results in Table I showed that the average 
Table I. Average domain sizes in sheared mesophase of the bisphenol copolyesters

\begin{tabular}{llll}
\hline $\begin{array}{c}\text { Polymer } \\
\text { sample }\end{array}$ & $\begin{array}{l}\text { Bridge } \\
\text { group }\end{array}$ & $\theta_{\max } /{ }^{\circ}$ & $L / \mu \mathrm{m}$ \\
\hline TIHBP & - & 4.068 & 13.56 \\
TIHBPM & $\mathrm{CH}_{2}$ & 3.950 & 14.03 \\
TIHSDP & $\mathrm{SO}_{2}$ & 3.678 & 15.06 \\
TIHBPA & ${\mathrm{C}\left(\mathrm{CH}_{3}\right)_{2}}$ & 3.134 & 17.67 \\
\hline
\end{tabular}

domain sizes of the copolyesters followed the trend: TIHBPA > TIHSDP > TIHBPM > TIHBP, which was in agreement with the trend of steric effect of the bridge groups in bisphenols: $\mathrm{C}\left(\mathrm{CH}_{3}\right)_{2}>\mathrm{SO}_{2}>\mathrm{CH}_{2}>-$. The angle between two phenyl rings in the bisphenols was a critical factor determining the domain spacing. For BP, the angle was $180^{\circ}$ while for BPM, the angle was $110-111^{\circ}$. The angle became further small when the bridge groups in bisphenols were $\mathrm{SO}_{2}(\mathrm{SDP})$ and $\mathrm{C}\left(\mathrm{CH}_{3}\right)_{2}$ (BPA) due to steric effect. For BPA, the two aromatic rings were twisted relatively around each other rather than forming a shallow $\mathrm{V}$ shape as did the others. ${ }^{19}$ The steric effect was also responsible for the liquid crystalline behaviors of the copolyesters. The light intensity under crossed polarizers in Figure 3 indicated that optical anisotropy followed the trend TIHBPA $<$ TIHSDP $<$ TIHBPM $<$ TIHBP. It was because that the liquid crystalline behavior, or the optical anisotropy in the mesophase increased with increasing chain linearity. ${ }^{1}$

\section{REFERENCES}

1. R. W. Lenz and J.-I. Jin, Macromolecules, 14, 1405 (1981).

2. S. R. Rojstaczer and R. S. Stein, Macromolecules, 23, 4863 (1990).

3. B. S. Hsiao, R. S. Stein, K. Deutscher, and H. H. Winter, J. Polym. Sci., B, Polym. Phys., 28, 1571 (1990).

4. G. C. Berry, Mol. Cryst. Liq. Cryst., 165, 333 (1988).

5. B. Ernst and P. Navard, Macromolecules, 22, 960 (1989).

6. Z. Zhao, X. Wu, Y. Lin, and J. McLean, Polym. Bull., 31, 151 (1993).

7. Z. Zhao, X. Wu, Y. Lin, and J. McLean, accepted for publication in J. Appl. Polym. Sci.

8. R. Silvestri and L. L. Chapoy, Polymer, 33, 2891 (1992).

9. M. Glotin and L. Mandelkern, Macromolecules, 14, 1394 (1981).

10. J. Maxfield and L. Mandelkern, Macromolecules, 10, 1141 (1977).

11. Y. Li, J. Liu, H. Yang, D. Ma, and B. Chu, J. Polym. Sci., B, Polym. Phys., 31, 853 (1993).

12. T. Hashimoto, Y. Murakami, and H. Kawai, $J$. Polym. Sci., B, Polym. Phys., 13, 1613 (1975).

13. J. Aarten, J. Eur. Polym. J., 6, 1095 (1960).

14. M. B. Rhodes and R. S. Stein, J. Polym. Sci., A-2, 7, 1539 (1969).

15. E. P. Chang and J. S. C. W. Chien, Macromolecules, 5, 610 (1972).

16. R. E. Prud'homme and R. S. Stein, J. Polym. Sci., 12, 1805 (1974).

17. M. Moritani, N. Hayashi, A. Utsuo, and H. Kawai, Polym. J., 2, 74 (1971).

18. T. Hashimoto, A. Nakai, T. Shiwaku, H. Hasegawa, S. Rojstaczer, and R. S. Stein, Macromolecules, 22, 422 (1989).

19. G. W. Calundann and M. Jaffe, "Anisotropic Polymers, Their Synthesis and Properties," in Proceedings of the Robert A. Welch Conference on Chemical Research, XXVI. Synthetic Polymers, 1982. 